\title{
INDOORTUBES A NOVEL DESIGN FOR INDOOR MAPS
}

\author{
A.S. Nossum ${ }^{a}$ \\ a Norwegian University of Science and Technology, N-7491 Trondheim, NORWAY - alexander.nossum@ntnu.no
}

KEY WORDS: Indoortubes, underground maps, indoor maps, cartography

\begin{abstract}
:
Efforts within cartography on indoor maps have previously not received a lot of attention. Work that has been carried out on indoor maps often focus on map design very similar to an architectural style (Klippel et al. 2006, Ciavarella and Paternò 2004). In some cases the design has been of a more novel character where approaches with augmented and virtual realities have been carried out (Radoczky 2007, Müller et al. 2006). Common to these approaches is the depiction of one floor per map. As well, the primary user task is often solely personal navigation. In this article we present an innovative approach to indoor maps. The design is directly inspired by underground tube maps first developed by Harry Beck (Garland 1994) and today a common design for public transport maps. The main advantages of the map design are its simplicity and the possibility of including all floors in one map view. This allows the map user to easier comprehend the structure of the building without using several maps, as commonly needed with today's indoor map designs. We present two different map styles each intended to satisfy different user groups and tasks depending on the user's familiarity with the environment. In addition we motivate for new application areas suitable for indoor maps, especially large hospitals. We hypothesize that the design proposed in this article leans very well to displaying real-time dynamic geospatial information, such as patients, staff, equipment and room availability within hospitals. Due to the early phase of the work presented here we outline the needed further work and possibilities of technological platforms as well as evaluations necessary in order for the design to gain acceptance and success.
\end{abstract}

\section{BACKGROUND}

In cartography much effort has been put into the study and analysis of the human perception of maps. This result in guidelines and different map styles which inherits different qualities suitable for different purposes, ranging from abstract maps to close to real life 3-dimensional augmented realities. Similar to mostly all of these efforts is the focus of the depiction of outdoor environments.

Maps focusing on indoor environments, such as large buildings, rarely receive attention in the cartographic community. Indoor maps are commonly used for emergency and evacuation maps. Typically found in corridors in large and public buildings. The standard style of these maps is very close to architectural maps used for construction, where one floor is depicted from above in a 2dimensional fashion. The cartography generally receives little attention. Typically the orientation is not egocentric and the level of detail is very high resulting in a visual clutter and problems of finding the nearest exit fast.

Earlier work has addressed the cartography in emergency maps to some degree. Klippel et al. (2006) propose a set of general guidelines for "you-are-here-maps" (YAH maps). The guidelines are of a general kind and are suitable for evaluating the quality of already existing "you-are-here" maps. However, the guidelines are not directly applicable for other purpose indoor maps, or for the design of new maps. Additionally the architectural style of the maps seems to be accepted without discussion of alternative visual representations.
Radoczky (2007) discusses several visual representation methods for indoor maps and their different qualities. The traditional architectural style maps, named floor plans, are argued to be one of the better suited representation methods for indoor environments. However, 3-dimensional representation, such as virtual environments, is argued to be the best; although, the technological capabilities for this were not ready at the time of the writing (Radoczky 2007). The technological situation is different today, especially for mobile devices with medium sized high-resolution screens and enough computational power capable of displaying 3dimensional virtual realities. However, it is not certain that virtual realities are good for all purposes in indoor environments. Virtual realities are often made to resemble the reality as close as possible. One of the strengths of traditional outdoor maps is the ability to get fast overview over large areas due to abstract representation of the environment. Virtual realities do not exhibit this kind of abstract representation and gaining fast overview will inevitably be harder for the user. This argues for the development of new visual representation methods for indoor maps.

Some prototypes using indoor maps have been made and tested out for different purposes in indoor environments. Müller et al. (2006) presents a mobile indoor navigation system. The system interacts with large printed floor plan maps positioned at important locations within the building. Thus, the mobile system never displays the map itself, but augments the path on top of an image of the printed map. This can be said to be an early variant of augmented reality. The cartography is not discussed thoroughly, and as

A special joint symposium of ISPRS Technical Commission IV \& AutoCarto

in conjunction with

ASPRS/CaGIS 2010 Fall Specialty Conference

November 15-19, 2010 Orlando, Florida 
mentioned a standard floor plan map is used as map style. Issues of orientation, overview and not least navigation across floors will inevitably occur.

Ciavarella and Paternò (2004) present a similar mobile system intended as a museum guide. The system reacts to the location of the user and displays information and a map of the current location. The map design is an architectural style floor map in two different variants, one detailed and one intended for overview. From a user evaluation the authors found that egocentric maps are preferred, as well the users gave feedback that the map design should be changed.

The earlier work briefly presented here indicates that the chosen map style for indoor maps are often basic floor plans. We argue that this map style is not the best at depicting user friendly indoor maps. Specifically mentioned are issues of easy perception of several floors, overview of one or several floors, level of detail, and the general perception of these kinds of maps.

Technological capabilities have increased tremendously since the early prototypes discussed here were implemented. Off-the-shelf mobile devices are now capable of storing and computing large amount of data as well as containing high-resolution screens. This argues for the study and development of new innovations focusing on indoor cartography and maps suitable for new usage scenarios.

\section{APPLICATION AREAS}

Earlier work has primarily focused on indoor maps for personal navigation (Klippel et al 2006, Radoczky 2007, Müller et al. 2006, Ciavarella and Paternò). Mobile systems with small screens, low resolution and computational capabilities have been a preferred technological platform. As mentioned earlier, the map style for these systems has not been discussed nor have alternatives been properly suggested. We strongly believe that the application area of indoor maps spans wider than solely personal navigation. With the technological capabilities of today several of the issues of the previous mentioned prototypes are now solved. In addition, users of modern mobile systems are now requiring real-time location and user specific information with high degree of interactivity. This suggests that the traditional approach of floor plans with route information does not meet the requirements of today, nor do they fulfil the potential of today's technology.

A technological issue that is still present, and of concern, is the lack of proper and accepted indoor positioning systems. Several attempts on indoor positioning have been made. The most successful and promising technologies seems to be the use of WiFi (Moen and Jelle 2007, Schrooyen et al. 2006, Fry and Lenert 2005, Muñoz et al. 2003), ultrasound (Mautz 2009) and the new Galileo system (Yang et al. 2007). Based on this we believe that indoor positioning will be available in the near future.
Although indoor positioning is not currently available apart from specific laboratory settings, we strongly believe that the work on cartography for indoor maps should be in advance and ready to meet the technological advances. This will inevitably make the acceptance and implementation of scientifically funded results easier when indoor positioning is mature and implemented. Additionally, indoor maps can successfully exist without the need for indoor positioning, as proven by today's emergency maps.

One promising application area for indoor maps is large hospitals. Large hospitals are complex buildings with a highly dynamic environment. A large number of patients, staff and equipment need to be coordinated and scheduled in order to make the process as fluid as possible. In addition, the different rooms have different facilities and schedules, such as operating rooms, emergency rooms and similar. Scheduling and planning in advance is often not feasible due to the rapid changing nature of a hospital. This is especially visible in emergency situations where for instance scheduled surgeries are postponed in favour of more emergent surgeries. This makes coordination work highly demanding.

The location of all involved actors, equipment and rooms is believed to be an enabling factor supporting the coordination work (Marjamaa et al. 2006). For coordination workers, the location is especially important in order to get awareness and overview of the current situation. Situational awareness for non-coordinators is also believed to be enabling for the self-coordination of the regular staff at hospitals (Fry and Lenert 2005).

Traditional outdoor maps have proven to be highly successful at coordination and overview over large and complex environments. However, indoor environments open new issues with potentially very high information density and especially the added complexity of multiple floors. The latter adds a new dimension to the environment with the need to visually represent discreet floors in the third dimension.

In addition to the complexity of the environment, indoor maps for hospitals are faced with the issue of dealing with a large variety in potential users. There is a high probability that the regular staff is known to the geography and knows where the rooms and equipment is, or should be, situated. Patients and visitors on the other hand are unknown to the geography and should not have access to certain information, such as patient sensitive information. Indoor maps needs to not only visually represent the environment, but also consider the different user groups found in the environment.

Large, public, whiteboards have been successfully used in hospitals for increasing the situational awareness of staff (Bardram and Bossen 2005, Bossen 2002). The information is typically of a textual and symbolic kind and also including location to a certain degree. Large screens displaying indoor maps illustrating the current location and potentially the state of different actors, equipment and rooms is believed to be very successful in order to more visually and rapidly support the situational awareness as

A special joint symposium of ISPRS Technical Commission IV \& AutoCarto

in conjunction with

ASPRS/CaGIS 2010 Fall Specialty Conference

November 15-19, 2010 Orlando, Florida 
well as enable easier coordination between the workers. For instance in addition to large whiteboards or digital whiteboards.

\section{INDOOR TUBE MAPS}

Harry Beck introduced a new way of portraying the London metro system in the 1930's. The most revolutionary aspect of Beck's tube map was the negligence of the geographic accuracy (Garland 1994). The topological ordering of the stations was however retained. This allowed for more freedom in the visual positioning of the stations and lines to make the map more visually pleasing and easier to perceive. Earlier tube maps were geographically correct making the lines and stations visually cluttered and seemingly unordered (Garland 1994). In addition to the visual layout of the lines the tube map consists of large amounts of information, as there are a lot of stations, each with a name, and a lot of overlapping and parallel lines, either physical or non-physical.

The map style developed by Beck was inspired by electrical diagrams. These kinds of diagrams are visually pleasing to look at due to their regularity. The bends of the curves are important in achieving this regularity. They are only bent at certain angles, a part from straight, where 45 and 60 degrees is the most common. Colour and texture is used to differentiate between the different lines. Stations occur in primarily three different fashions; small stations, large stations and connecting stations. Small stations are represented using a marker orthogonal to the line. Larger and connecting stations are represented using circles which are connected for connecting stations. In modern tube maps station names are included as text adjacent to the station. Combined this makes the information density quite high especially when considering that there are several overlapping lines with potentially different stops.

The issues faced by mapping the metro system are similar challenges faced by mapping indoor environments. Rooms occur as both minor and major, such as private offices, meeting rooms and similar. All rooms are connected by corridors or other walking areas. Corridors are overlapping across floors, and each floor is connected through elevators and stairs. Combined this makes the information density similarly high as with the metro system. In addition to this, the geographic accuracy is hypothesized to be of little importance for indoor environments in relation to the topological accuracy. It is, however, believed that the geographic accuracy is more important for indoor maps than for underground metro maps. The distance and orientation in indoor environments should be more or less correct due to the navigation and orientation of the user.

Inspired by the similarities identified above, we propose a new map style for indoor maps; indoor tube maps. Indoor tube maps are directly inspired by modern maps for underground metro systems. We propose two different views of an indoor tube map. One is a flat 2-dimensional view, very similar to underground maps. The third dimension is collapsed in favour of easy overview of the complete information and the building. The other view is a perspective view where the different floors are in separate layers and positioned in three dimensions - equal to the way the floors are positioned in real life.

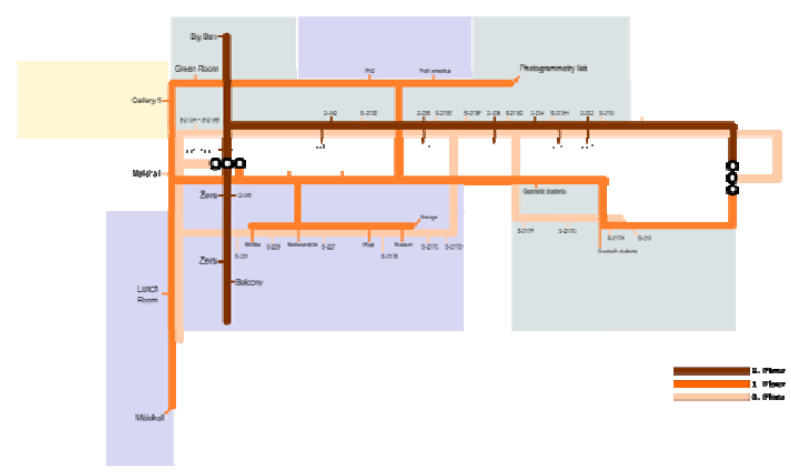

Figure 1. Variant of 2-dimensional view with overlapping lines and highlighted general areas ${ }^{\dagger}$

The general grammar of the indoor tube maps are that corridors are represented as lines, similar to the railway lines in underground maps. Currently only two room types are represented, small or private rooms and large or public rooms. All rooms have the possibility of labels with an identifiable text, such as the room number, the name of the person located there or similar. Connected circles act as symbols for elevators and stairs, which connect floors together - parallel with connecting stations which connect different lines in underground maps.

Figure 1 illustrates one version of the 2-dimensional view. The style is very similar to the style of underground maps. All corridors are collapsed into the map regardless of the floor they are at. The relationship between the floors is subtly indicated where the corridors overlap, the darkest floor is for instance on top of the brighter floors. The symbols representing rooms are small markers. The label size indicates the importance or the size of the room - for instance for public rooms. Additional emphasis is put on the current floor by highlighting overviews of different areas of the floor such as office areas, student areas and similar. This is believed to strongly aid the user's orientation and perception of the floor hierarchy. It is believed that the colouring of the floors and general areas of the current floor is easily learned and remembered which makes this type of map suitable for regular users which know the environment, but for instance requires additional spatial information.

\footnotetext{
${ }^{\dagger}$ Figures available at http://geomatikk.ntnu.no/projects/indoortubes
}

A special joint symposium of ISPRS Technical Commission IV \& AutoCarto
i n c o n j u n c t i o n w i t h
ASPRS/CaGIS 2010 Fall Specialty Conference

November 15-19, 2010 Orlando, Florida 


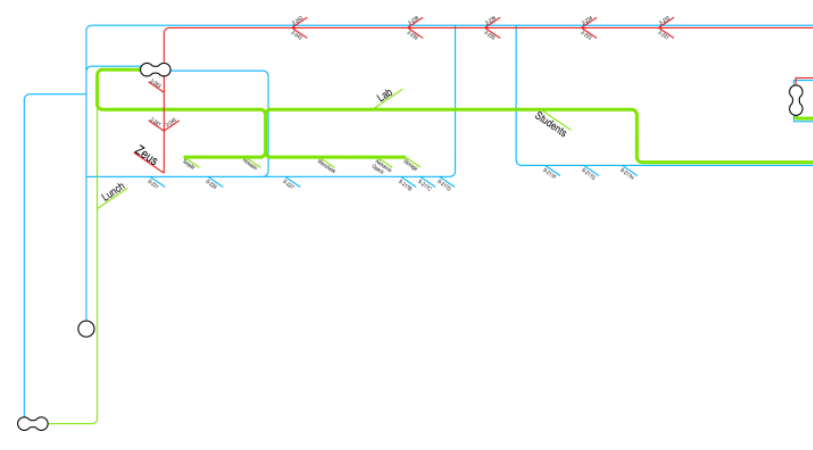

Figure 2. Segment of fishbone layout covering three floors ${ }^{\dagger}$

Figure 2 illustrates another version of the 2-dimensional view. The main differences are the markers for the rooms and their labels. The labels are fishbone-like with and angular line containing the text. This is an experimental version which differs slightly from the traditional underground maps and thorough evaluation of the differences in perception and usability needs to be performed in order to assess which is better in different contexts.

It is hypothesized that the 2-dimensional view is particularly suitable for tasks involving experienced users familiar with the general environment, such as regular staff or coordinators at a hospital. Tasks which require the user to get immediate overview of the current situation either at the current floor or across floors are ideal for this kind of map. This makes it also suitable for supporting implicit situational awareness due to the simplicity of the design, which is hypothesized to require minimal cognitive efforts when the user is familiar with it. The simplicity of the design additionally makes the map suitable for complex environments spanning several floors as each floor, as a minimum, requires only the corridor to be represented. Further exploration of the 2-dimensional map capabilities should consider testing the performance of the map for navigation tasks on users not familiar with the environment, which would give indications about the orientation and navigation capabilities as well.

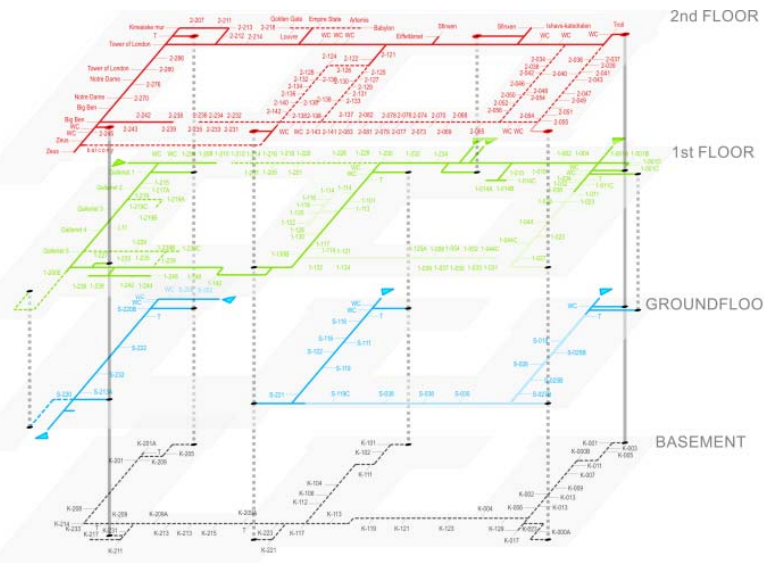

Figure 3. 3D perspective layout covering four floors. ${ }^{\dagger}$

The perspective view is intended to be more intuitive and direct representation of the building. An example map is found in figure 3 . The floors follow the same core principles as the 2-dimensional view; however the different floors are placed in layers and positioned on top of each other. In addition, the connecting stations metaphor has been replaced with vertical lines representing elevators and stairs. This makes the floors appear more equal to the reality their representing. The hypothesis is that the perspective or potential 3-dimensional view makes it easier for the user to perceive and understand the layout of the building and the different floors. Thus, this view is intended to be suitable for users not familiar with the environment. Navigation and exploratory tasks are both hypothesized to be well supported by this map style. However, it is believed that incorporating real-time information, such as moving actors, room states and similar are not suitable in this representation. This is believed to visually clutter the map and make it harder to perceive the information of interest.

In finished implementations of the perspective view, it is natural to consider the map layers to be contained in a virtual 3D environment, similar to the interface of virtual globes like Google Earth. This will make the map itself more interactive as it allows the user to navigate within floors and potentially view a virtual tour of important routes or similar. It is also envisaged that this perspective view could be suitable for incorporation in augmented reality systems for medium sized mobile screens. However both of these implementations will need further exploration, especially their performance relating to usability and perception for the intended user groups.

\section{FURTHER WORK}

In this paper we have motivated for new and innovative development and use of indoor maps in the field of cartography. The application of indoor maps in hospitals and large enterprises has been especially focused as an innovative and promising application area. Tasks like realtime maps for scheduling, overview and situational awareness are of particular interest.

Two different map styles have been suggested, both inspired by underground metro maps. One 2-dimensional style focused in particular on overview tasks suitable for users familiar with the environment. The other, a 3dimensional perspective style with a more intuitive representation of the relationship between the different floor plans. The latter style suitable for non-familiar users with tasks like personal navigation and gaining familiarity with the environment.

Both of the proposed indoor map styles are in a very early development phase. Refinement of the graphical style is most certainly needed in addition to further investigations into the applicability of the maps. In addition to the graphical style further work needs to investigate and explore the technological possibilities of these kinds of maps. Mobile devices are natural platforms to investigate

A special joint symposium of ISPRS Technical Commission IV \& AutoCarto

in conjunction with

ASPRS/CaGIS 2010 Fall Specialty Conference

November 15-19, 2010 Orlando, Florida 
further with the medium sized screens as well as good communication possibilities with wireless network and large storage capabilities. Screen size will inevitably affect the map design. Concerns have to be taken into both the information density as well as the graphical span of the map itself. Interactivity of indoor maps on mobile devices is also of concern. It is most surely needed some form of interactivity in order to navigate the maps, however what kind of interactivity suits the different users best is still unknown. Further efforts should focus not only on the application of traditional interactivity methods, but also include the recent multi-touch and tactual interfaces now possible on consumer products like smart-mobiles and tablet pc.

The successful application of whiteboards in hospitals (Bardram and Bossen 2005, Bossen 2002) briefly mentioned earlier suggests that large screens are appropriate to investigate within hospital contexts. Large screens can also enable interactivity with the users similar to what whiteboards do today. However, we believe that the successful interactive methods for mobile devices do not necessarily apply directly on large, fixed screens. Multitouch interfaces for large screens are natural to investigate. However, non-physical interfaces are also of interest, such as pointers and similar. Further work will need to investigate and develop new knowledge of different interactivity possibilities of indoor maps for both medium and large screens.

It is suggested that the further work consider screen size and interactivity, but more importantly consider the potential users, which are going to use the maps, carefully and includes the users in the design phase. Intermediate user evaluations of the results are needed in order to get successful and accepted maps for indoor environments. Hospitals are believed to be one domain where the need for indoor maps is rising. Additionally, the indoor hospital environment leans very well to development of complex indoor maps. We are currently in the process of working toward the refinement of the proposed map styles as well as work on interactivity methods, briefly mentioned above.

\section{REFERENCES}

J. E. Bardram \& C. Bossen 2005. 'A web of coordinative artifacts: collaborative work at a hospital ward'. In GROUP '05: Proceedings of the 2005 international ACM SIGGROUP conference on Supporting group work, pp. 168-176, New York, NY, USA. ACM.

C. Bossen 2002. 'The parameters of common information spaces: the heterogeneity of cooperative work at a hospital ward'. In CSCW '02: Proceedings of the 2002 ACM conference on Computer supported cooperative work, pp. 176-185, New York, NY, USA. ACM.

C. Ciavarella \& F. Paternò 2004. 'The design of a handheld, location-aware guide for indoor environments'. Personal and Ubiquitous Computing 8(2):82-91.

A special joint symposium of ISPRS Technical Commission IV \& AutoCarto

in conjunction with

ASPRS/CaGIS 2010 Fall Specialty Conference

November 15-19, 2010 Orlando, Florida Symposium pp. 261-265.
E. A. Fry \& L. A. Lenert 2005. 'MASCAL: RFID tracking of patients, staff and equipment to enhance hospital response to mass casualty events.'. AMIA ... Annual Symposium proceedings / AMIA Symposium. AMIA

K. Garland 1994. Mr. Beck’s Underground Map: a history. Capital Transport Publishing.

A. Klippel, et al. 2006. 'You-Are-Here Maps in Emergencies The Danger of Getting Lost'. Journal of Spatial Science pp. 117-131.

R. A. Marjamaa, et al. 2006. 'Time Accuracy of a Radio Frequency Identification Patient Tracking System for Recording Operating Room Timestamps'. Anesthesia \& Analgesia 102(4):1183-1186.

R. Mautz 2009. 'Overview of Current Indoor Positioning Systems'. Geodesy and Cartography 35(1):18-22.

H. L. Moen \& T. Jelle 2007. 'The Potential for LocationBased Services with Wi-Fi RFID Tags in Citywide Wireless Networks'. Wireless Communication Systems, 2007. ISWCS 2007. $4^{\text {th }}$ International Symposium on, pp. 148-152.

M. A. Muñoz, et al. 2003. 'Supporting Context-Aware Collaboration in a Hospital: An Ethnographic Informed Design'. pp. 330-344.

H. J. Müller, et al. 2006. 'Mobile Map InteractionEvaluation in an indoor scenario'. GI Jahrestagung 2:403410.

V. Radoczky 2007. 'How to design a pedestrian navigation system for indoor and outdoor environments'. pp. 301-316.

F. Schrooyen, et al. 2006. 'Real Time Location System over WiFi in a Healthcare Environment'. Journal on Information Technology in Healthcare 4(6):401-416.

B. Yang, et al. 2007. 'Indoor positioning performance of Galileo signal'. In Society of Photo-Optical Instrumentation Engineers (SPIE) Conference Series, vol. 6795 of Presented at the Society of Photo-Optical Instrumentation Engineers (SPIE) Conference. 